FORMATION Formation emploi

Revue française de sciences sociales

98 | avril-juin 2007

Pour une approche par les capacités

\title{
Les dérives de la formation professionnelle
}

Pierre Cahuc et André Zylberberg

\section{(2) OpenEdition}

\section{Journals}

Édition électronique

URL : http://journals.openedition.org/formationemploi/1639

DOI : 10.4000/formationemploi.1639

ISSN : 2107-0946

Éditeur

La Documentation française

Édition imprimée

Date de publication : 1 avril 2007

Pagination : 155-156

ISSN : 0759-6340

\section{Référence électronique}

Pierre Cahuc et André Zylberberg, «Les dérives de la formation professionnelle », Formation emploi [En ligne], 98 | avril-juin 2007, mis en ligne le 30 juin 2009, consulté le 30 octobre 2020. URL : http:// journals.openedition.org/formationemploi/1639; DOI : https://doi.org/10.4000/formationemploi.1639

(c) Tous droits réservés 


\title{
Débat
}

\section{Les dérives de la formation professionnelle}

\author{
Par Pierre Cahuc et André Zylberberg*
}

\begin{abstract}
Pierre Cahuc et André Zylberberg proposent une synthèse des critiques du système français de formation professionnelle, issue de leur rapport intitulé "La formation professionnelle des adultes : un système à la dérive »?
\end{abstract}

Face à l'accélération du progrès technique, à l'intensification de la concurrence internationale, à l'allongement de la durée de vie, la formation professionnelle continue constitue aujourd'hui un instrument essentiel pour assurer la cohésion sociale, la sécurisation des parcours professionnels et de bonnes performances macroéconomiques. Forte de ce constat, la loi Fillon, votée en mai 2004, a institué le Droit individuel à la formation, le désormais célèbre «DIF », qui offre la possibilité à tout salarié d'accéder à 20 heures de formation par an cumulables pendant 6 ans. Par ailleurs, le seuil obligatoire de dépenses pour la formation continue a crû significativement : il est passé de $1,5 \%$ à $1,6 \%$ de la masse salariale dans les entreprises de 10 salariés ou plus et de $0,25 \%$ à $0,55 \%$ dans les autres. Au total, en 2005, la France a dépensé 23 milliards d'euros, soit 1,4\% du PIB (produit intérieur brut), en actions de formation professionnelle continue, soit environ trois fois le montant des dépenses pour le RMI (revenu minimum d'insertion).

En règle générale, il faut se féliciter de l'introduction d'un nouveau «droit». A priori, plus de droit signifie plus de transparence et moins d'inégalités.

${ }^{1}$ Centre d'observation économique de la chambre de commerce et d'industrie de Paris, Juillet 2006, 73 p.
En outre, le DIF résulte d'un consensus auquel ont adhéré tous les partenaires sociaux. Le DIF est en effet la transcription directe dans la loi de 2004 d'un Accord national interprofessionnel signé en 2003. On n'avait pas vu une telle unanimité sur un sujet d'importance depuis la loi Delors de 1971 instaurant la formation professionnelle.

Hélas, nous craignons qu'il faille rapidement déchanter. Ce n'est pas en ajoutant un dispositif supplémentaire, fut-il unanimement célébré par les partenaires sociaux, que notre système de formation professionnelle deviendra nécessairement plus juste et plus efficace. En réalité, le DIF risque d'accroître

* Pierre Cahuc est professeur à l'université Paris 1, chercheur associé au Crest (Centre de recherche en économie et statistique)-Insee, à l'IZA (Institut for the Study of Labor) et au CEPR (Centre for Economic Policy Research).

André Zylberberg est directeur de recherche au Cnrs, membre du Centre d'économie de la Sorbonne de l'université Paris 1, membre de l'École d'économie de Paris. Leurs domaines de recherche sont l'économie du travail et les politiques d'emploi. Ils ont publié récemment ensemble Le chômage, fatalité ou nécessité ? Flammarion, collection "Champs », 2005 ; Labor Economics, MIT Press, 2004. 
les dysfonctionnements d'un système mal conçu car on peut légitimement craindre que ce nouveau «droit» creuse un peu plus les inégalités et augmente les financements opaques, mal contrôlés par les pouvoirs publics, qui entravent l'efficacité de notre système de formation professionnelle.

Les très nombreuses évaluations menées dans un grand nombre de pays montrent que les dispositifs indifférenciés, de courte durée, ouverts à tous, mais où tous n'ont ni les mêmes besoins ni les mêmes capacités, ne font qu'accroître les inégalités existantes : les mieux lotis au départ en tirent beaucoup d'avantages ; en revanche, les plus éloignés de l'emploi n'en bénéficient que rarement. Ainsi, dans un système qui se limite à offrir les mêmes droits formels à tous, les salariés les plus défavorisés se forment peu, tout en contribuant à payer les stages des cadres travaillant dans les grandes entreprises. Pour jouer un véritable rôle de promotion sociale, la formation professionnelle doit offrir des formations longues, et donc coûteuses, à des publics ciblés de façon restreinte. Si le but recherché était effectivement d'améliorer le sort des personnes les plus vulnérables, le DIF, en obligeant toutes les entreprises à fournir de façon indifférenciée une formation courte à tous les salariés, est donc une stratégie vouée à l'échec.

La hausse du seuil obligatoire des dépenses de formation professionnelle ne devrait pas non plus contribuer à faire régresser l'opacité du financement de l'ensemble du dispositif. La collecte des fonds correspondant à ces obligations légales est l'apanage d'Organismes paritaires collecteurs agréés (OPCA). À charge pour eux de les mutualiser et de les redistribuer selon les besoins. Ces organismes sont gérés de manière paritaire par des représentants des syndicats de salariés et patronaux et engrangent une recette annuelle de plus de 4 milliards d'euros. Pourtant, l'efficacité réelle des dépenses de ces organismes n'est jamais évaluée. Certes, $1,5 \%$ de ces dépenses servent très officiellement à financer les organisations syndicales représentatives, mais il est difficile d'y voir une saine manière de financer le paritarisme. Celui-ci aurait tout à gagner à plus de transparence.

En réalité, l'introduction du DIF a tendance à amplifier les défauts inhérents au principe «former ou payer », clef de voûte de notre système et exception française (il semblerait que seule la province du
Québec l'ait expérimenté... puis abandonné), qui contraint les entreprises à dépenser une fraction minimale de leur masse salariale pour la formation professionnelle. Ce principe a deux défauts majeurs. Tout d'abord, il n'incite pas les très nombreuses entreprises qui dépassent le seuil minimum à accroître leur effort de formation puisqu'elles doivent supporter la totalité du coût des formations dispensées au-delà de ce seuil. Ensuite, une grande partie des ressources sont prélevées par les OPCA censés orienter les dépenses vers les formations les plus efficaces. En fait, ce système confère à ces organismes un pouvoir exorbitant dans l'orientation du marché de la formation professionnelle, qui entraîne de fortes distorsions de concurrence.

Ainsi, améliorer la formation continue des adultes ne passe pas aujourd'hui par un accroissement systématique de financements qui alimentent un système fondé sur de mauvais principes. De profondes réformes s'imposent; elles devraient s'appuyer sur les orientations suivantes :

1) l'obligation de «former ou payer » doit être abandonnée et remplacée par des prélèvements allant directement dans les caisses de l'État, puis reversés sous forme de subventions octroyées au prorata des dépenses de formation dont la qualité est certifiée par des organismes indépendants ;

2) le service public de l'emploi et de la formation professionnelle des adultes doit être réorganisé autour d'une agence offrant des parcours différenciés aux demandeurs d'emploi et qui s'appuie sur des opérateurs externes, mis en concurrence et rémunérés en fonction des succès obtenus dans le retour vers l'emploi des chômeurs ;

3) il faut repenser le dispositif indifférencié du DIF et réorienter les dépenses, en offrant aux personnes en difficulté des formations longues, intensives et ancrées dans le secteur marchand.

Rares sont les acteurs de notre système de formation professionnelle qui contestent l'ampleur des dysfonctionnements, mais encore plus rares sont ceux qui se disent prêts à changer les règles du jeu, tant les enjeux financiers figent les positions. Pourtant, faute de réformes radicales, la formation professionnelle à la française continuera à dériver avec le risque de sombrer dans un naufrage coûteux. 